\title{
El papel protector de las variables psicológicas positivas y su relación con el cambio clínico tras la intervención en los trastornos adaptativos
}

\author{
Silvia Fuentes Cerdá \\ al287715@uji.es \\ IRYNA RACHYLA \\ rachyla@uji.es \\ SONIA MOR RODRÍGUEZ \\ al189465@uji.es \\ MAR MOLÉs AmpostA \\ molesm@uji.es \\ SoledAd Quero CASTELLANO \\ squero@uji.es
}

\section{Resumen}

Introducción: El trastorno adaptativo (TA) es uno de los problemas psicológicos más prevalentes, pero se desconocen los factores que puedan influir en su gravedad o respuesta al tratamiento. Diferentes estudios indican que el bienestar y otras variables positivas pueden influir en la morbilidad y cambio clínico (CC). Objetivos: Analizar la capacidad de algunas variables psicológicas positivas (VP+) para predecir la intensidad de la sintomatología clínica en pacientes con TA, y explorar en qué medida predicen el CC tras la intervención. Método: La muestra incluyó 44 pacientes. Los cuestionarios de autoestima, refuerzos ambientales (EROS) y bienestar psicológico (BP-29) se utilizaron como medidas de VP+ antes del tratamiento psicológico. El CC en síntomas de depresión, estrés y pérdida, y afecto positivo y negativo se evaluó en el posttratamiento y seguimientos (3, 6 y 12 meses). Resultados: Un entorno reforzante predecía la intensidad de la sintomatología clínica, siendo ésta menos grave en pacientes que lo valoraban como más reforzante. Poseer en menor medida VP+ relacionadas con autoestima, refuerzo del entorno y ciertas dimensiones de bienestar psicológico (crecimiento personal y autoaceptación), predecía mayor CC después de la intervención. Conclusiones: Los resultados apoyan la hipótesis de que las VP+, tanto personales como del entorno, funcionan como factores protectores ante la adversidad en pacientes con TA. Sin embargo, el poseer en menor medida dichas cualidades parece no impedir a los pacientes beneficiarse del tratamiento. Esto podría indicar que durante la intervención los pacientes adquieren conocimientos y herramientas necesarias para el cambio, independientemente de sus recursos iniciales.

Palabras clave: Trastornos adaptativos, bienestar psicológico, autoestima, entorno reforzante y cambio clínico 


\section{Abstract}

Introduction: Adjustment Disorders (AD) are one of the most prevalent psychological problems, but the factors that might influence in their severity or response to treatment are unknown. Different studies indicate that well-being and other positive variables may influence morbidity and clinical change (CC). Objectives: To analyze the capacity of some positive psychological variables $(\mathrm{PV}+)$ to predict the intensity of the clinical symptoms in patients with $A D$, and to examine whether they predict the $C C$ after intervention. Method: The sample consisted of 44 patients. The questionnaires of self-esteem, environmental reinforcements (EROS) and well-being (BP-29) were used as measures of $\mathrm{PV}+$ before receiving psychological treatment. The CC in symptoms of depression, stress and loss, and positive and negative affect were assessed at post-treatment and follow-ups (3, 6 and 12 months). Results: A reinforcing environment predicted the intensity of the clinical symptoms, being this less severe in patients who perceived it more reinforcing. Having in a lesser extent PV+ related to self-esteem, environmental reinforcement and certain dimensions of well-being (personal growth and self-acceptance) predicted greater CC after intervention. Conclusions: Results support the hypothesis that $\mathrm{PV}+$, both personal and environmental, act as protective factors against adversity in patients with $A D$. However, having in a lesser extent these qualities seems not to prevent patients to get benefit from treatment. This could indicate that during the treatment patients acquire the knowledge and tools needed for the change, regardless their initial resources.

Keywords: Adjustment disorders, psychological well-being, self-esteem, environmental reinforcement and clinical change

\section{Introducción}

El Trastorno Adaptativo (TA) se define, según el DSM-5 (APA, 2013), como una reacción emocional y/o comportamental negativa que interfiere en distintas áreas de la vida de la persona y aparece en respuesta a un acontecimiento estresante identificable dentro de los 3 meses siguientes a la aparición de éste.

EI TA es uno de los problemas psicológicos más frecuentes en los ámbitos de atención primaria y hospitalaria (Carta, Balestrieri, Murru, y Hardoy, 2009). Se trata de un importante problema de salud que tiene un gran impacto negativo en la persona y se asocia a un notable deterioro en las áreas social y laboral, causando un alto porcentaje de bajas laborales (Calvo, 2009). Además, se trata de un trastorno en el que existe un alto riesgo de suicidio (Cassey, Jabbar, O'Leary, y Doherty, 2015). Sin embargo, pese a la gran relevancia de los TA en cuanto al sufrimiento personal, gasto sanitario y pérdidas laborales, hay una notable escasez de trabajos existentes sobre su tratamiento (Casey Dowrick, y Wilkinson, 2001).

En los últimos años el interés y la investigación en el campo de Psicología Positiva ha aumentado considerablemente. En este sentido, se considera que una ciencia y práctica completas de la Psicología deben incluir la comprensión tanto del sufrimiento como de la felicidad, así como intervenciones dirigidas no únicamnete a aliviar el sufrimiento sino también a aumentar la felicidad. (Seligman, Steen, Park y Peterson, 2005). En este contexto, por un lado, hay estudios que demuestran que tener una mayor emocionalidad positiva aumenta la flexibilidad 
psicológica y refuerza los recursos físicos, intelectuales y sociales (Fredickson, 1998). Diferentes modelos teóricos sugieren que la emocionalidad positiva es un rasgo de personalidad estable y heredable (Krueger, McGue y lacono, 2001) y que, a bajos niveles, aumenta el riesgo de depresión y agrava el curso de la enfermedad (Clark, 2005; Clark y Watson, 1991; Davidson, 1998; Watson, Stasik, Ellickson-Larew, y Stanton, 2015). Así, las personas deprimidas presentan una tendencia menor a experimentar emociones positivas, incluso ante estímulos y situaciones positivas (Berenbaum y Oltmanns, 1992; Kaviani y cols., 2004; Klein, 1974; McFarland y Klein, 2009; Sloan, Strauss, y Wisner, 2001).

Por otro lado, se ha estudiado la relación existente entre variables psicológicas clínicas y variables psicológicas positivas (VP+), como el bienestar psicológico (Sheung-Tak y cols., 2017; Tseferidi, Griva, y Anagnostopoulos, 2017), el afecto positivo (Li, Starr, y Hershenberg, 2017; Charles, Lyubomirsky y Murray, 2017), la satisfacción con la vida (Küçük y Alemdar, 2017) y la autoestima (Johnson, Galambos, Finn, y Horne, 2017), indicando que estas variables pueden influir en el cambio clínico. Más concretamente, se ha observado que el afecto positivo funciona como amortiguador de la gravedad de la sintomatología clínica (Pressman y Cohen, 2012).

En el caso de los TA no se han encontrado en la literatura artículos que exploren la relación entre $\mathrm{VP}+$ y la gravedad y respuesta al tratamiento de los pacientes, lo que demuestra una vez más la escasez de estudios sobre estos trastornos pese a su gran relevancia. Por todo ello, los objetivos de este trabajo son, por un lado, analizar la capacidad de algunas VP+ para predecir la intensidad de la sintomatología clínica en pacientes con TA y, por otro, explorar en qué medida estas VP+ predicen el cambio clínico después de un tratamiento cognitivocomportamental (TCC).

\section{Método}

\section{Participantes}

La muestra estaba compuesta por 44 pacientes (33 mujeres y 11 hombres) diagnosticados de TA siguiendo los criterios del DSM-5 (APA, 2013) que habían solicitado ayuda en el Servicio de Asistencia Psicológica de la Universitat Jaume I de Castellón (España). La media de edad fue de 29.09 años (DT=8.95), con un rango que oscilaba entre 18 y 54 . La mayoría de la muestra $(75 \%)$ tenía estudios universitarios y el resto $(25 \%)$ estudios primarios y/o secundarios. Por lo que se refiere al subtipo de TA, la mayoría de los participantes $(70.5 \%)$ fueron diagnosticados de TA mixto con ansiedad y depresión, el 15.9\% tenían TA con estado de ánimo depresivo, el $11.4 \%$ con ansiedad y el $2.3 \%$ con alteración de emociones y de comportamiento. Por último, y con respecto al acontecimiento estresante sufrido, el $37.8 \%$ de la muestra presentaba TA debido a una ruptura/separación/divorcio, el $26.7 \%$ por problemas familiares, el $13.3 \%$ por cambio de residencia, el $12.2 \%$ por problemas laborales y el $6.7 \%$ a causa de un accidente.

\section{Medidas}

Medidas de variables positivas

Escala de Autoestima (Rosenberg, 1965): Se trata de un instrumento breve que permite explorar la autovaloración de uno mismo y los sentimientos de respeto y aceptación personal. 
La consistencia interna de la versión española hallada en el estudio de validación transcultural fue de 0.80 (Schmidt y Allik, 2005).

Bienestar Psicológico (BP-29: Ryff, 1989): Este instrumento proporciona información acerca del nivel de satisfacción de la persona en distintos ámbitos de su vida. La adaptación española del instrumento que fue utilizada en este estudio consta de 39 ítems y se compone de 6 subescalas (autoaceptación, relaciones positivas, autonomía, dominio del entorno, propósito en la vida y crecimiento personal), cuya consistencia interna va de 0.68 a 0.83 (Díaz y cols., 2006).

Environmental reward observation scale (EROS; Armento y Hopko, 2007): Este instrumento de 10 ítems ofrece una medida objetiva de lo reforzante que perceibe la persona su entorno. La adaptación española del instrumento (Barraca y Pérez-Álvarez, 2010) presenta una buena consistencia interna (0.86).

Medidas variables clínicas

Inventario de Depresión de Beck (BDI-Il; Beck, Steer y Brown, 1996): Este instrumento es uno de los más utilizados para evaluar la sintomatología depresiva. En este trabajo se utilizó la adaptación española realizada por Sanz, Navarro y Vázquez (2003) que muestra una elevada consistencia interna tanto para la población general (0.87) (Sanz, Perdigon, y Vázquez, 2003) como para la población clínica (0.89) (Sanz, García-Vera, Espinosa, Fortún, y Vázquez, 2005).

Inventario de Estrés y Pérdida (IEP; Quero, Mor, Molés, Rachyla, Baños, y Botella, en revisión): Este instrumento de 17 ítems permite evaluar el malestar que aparecen como consecuencia de vivir una situación difícil y/o estresante.. Datos preliminares de validación mostraron excelentes coeficientes de fiabilidad test-retest $(0.90)$ y de consistencia interna en población española tanto general (9.91) como clínica con TA (0.86) (Quero, Molés, Mor, Baños, y Botella, 2014).

Escala de Afecto Positivo y Afecto Negativo (PANAS; Watson, Clark, y Tellegen, 1988): Se trata de un cuestionario de 20 ítems que evalúa las dimensiones Positiva (p.ej., interesado, ilusionado, satisfecho) y Negativa del Afecto (p.ej., angustiado, culpable, agresivo). Cada ítem se responde en una escala de cinco puntos ( $1=$ Nada o muy ligeramente; $5=$ Mucho) indicando el grado en que cada adjetivo describe mejor el estado de ánimo del que responde. El instrumento presenta una alta consistencia interna y una buena fiabilidad test-retest (Watson y cols., 1988).

\section{Tratamiento}

Todos los participantes recibieron un TCC específico para los TA (Botella, Baños, y GuiIlén, 2008) que contaba con un número variable de sesiones (entre 6 y 8), dependiendo de las necesidades de cada paciente. Las sesiones tenían una duración de 90 minutos y se realizaban con periodicidad semanal. Los componentes terapéuticos incluidos fueron: psicoeducación; exposición; aceptación, afrontamiento y aprendizaje a partir de los problemas; y prevención de recaídas. También se incluyeron estrategias de Psicología Positiva con la finalidad de aumentar la resiliencia de los pacientes. Durante el procesamiento y elaboración del acontecimeintos estresante se utilizó el sistema de RV Ilamado El Mundo de EMMA, un ambiente virtual flexible que permite realizar una representación física de los significados y emociones que la persona asocia a diferentes situaciones problemáticas. Gracias a ello, es posible generar ambientes clínicamente significativos para cada participante, capaces de evocar acontecimientos estresantes diferentes (p.ej., ruptura sentimental, enfermedad, problemas laborales, etc.). Una descripción más detallada del Mundo de EMMA se puede encontrar en Quero y cols. (2017). 


\section{Procedimiento}

Después de ser incluidos en el estudio, y antes de recibir el tratamiento, los participantes completaron las medidas utilizadas en este trabajo. Una vez finalizada la intervención, se volvieron a medir las variables clínicas en el post-tratameinto y seguimiento de los 3, 6 y 12 meses. Diferencias entre las puntuaciones directas obtenidas en el pre-tratamiento y cada uno de los momentos de evaluación post-intervención fueron calculadas para obtener indicadores de cambio clínico en cada una de las medidas de variables cínicas (BDI-II, IEP y PANAS).

\section{Resultados}

Con el objetivo de analizar en qué grado las variables psicológicas positivas (VP+) predecían la intensidad de la sintomatología del TA, se llevó a cabo un análisis de regresión lineal por pasos tomando como variables independientes (VI) las medidas de las VP+ (autoestima, las 6 dimensiones de bienestar psicológico y el grado de refuerzo del entorno) y como variables dependientes (VD) las puntuaciones obtenidas en los instrumentos que evaluaban las variables clínicas (BDI, IEP y PANAS).

Como se puede observar en la Tabla 1, haber percibido el entorno como más reforzante predijo una menor intensidad en la sintomatología depresiva, sintomatología asociada al acontecimiento estresante, afecto negativo, así como, mayor afecto positivo.

Tabla 1

Resultados del análisis de regresión entre las VP+ y las variables clínicas evaluadas en el pre-tratamiento

\begin{tabular}{llcccc}
\hline PRE-tratamiento & & $\mathrm{R}^{2}$ & $\beta$ & $\mathrm{t}$ & $\rho$ \\
\hline BDI & EROS &, 22 & $-0,49$ & $-3,53$ &, $001^{* *}$ \\
IEP & EROS &, 13 & $-0,37$ & $-2,38$ &, $022^{* *}$ \\
PANAS + & EROS &, 34 & 0,59 & 4,60 &, $000^{* *}$ \\
PANAS - & EROS &, 19 & $-0,45$ & $-3,22$ &, $003^{* *}$ \\
\hline
\end{tabular}

${ }^{* *} \rho<0,01 ;{ }^{*} \rho<0,05$

Con el fin de explorar en qué medida poseer VP+ predecía la eficacia del tratamiento para el TA, también se realizó un análisis de regresión por pasos. Las medidas de las VP+ fueron utilizadas nuevamente como VI, mientras que los indicadores de cambio clínico en cada uno de los cuatro momentos de evaluación post-intervención (post-tratamiento, seguimiento de los 3, 6 y 12 meses) fueron utilizados como VD.

La Tabla 2 presenta los resultados referidos al cambio en el BDI-II. Como se puede observar, las personas que habían mostado una autoestima más baja antes de la intervención, presentaron una mayor mejoría en la sintomatología depresiva en el post-tratamiento y los seguimientos de los 3 y 12 meses. La misma relación se encontó entre EROS y el cambio en el BDI-II observado a los 6 meses de finalizar el tratamiento. Por último, la percepción de un mayor dominio del entorno fue predictor de un mayor cambio clínico en el BDI en el seguimiento a los 12 meses. 
Tabla 2

Resultados del análisis de regresión entre las VP+ y el cambio clínico en el BDI

\begin{tabular}{llcccc}
\hline BDI & & $\mathrm{R}^{2}$ & $\beta$ & $\mathrm{t}$ & $\rho$ \\
\hline Pre-post & Autoestima &, 14 & $-0,37$ & $-2,55$ &, $015^{*}$ \\
Pre-seg3 & Autoestima &, 34 & $-0,61$ & $-4,09$ &, $000^{* *}$ \\
Pre-seg6 & EROS &, 41 & $-0,66$ & $-4,34$ &, $000^{* *}$ \\
& Autoestima & & $-0,85$ & $-5,14$ &, $002^{* *}$ \\
Pre-seg12 & BP-29: Dominio del entorno &, 79 & 0,50 & 3,06 &, $002^{* *}$ \\
\hline
\end{tabular}

${ }^{* *} \rho<0,01 ;{ }^{*} \rho<0,05$

En cuanto a la gravedad de la sintomatología clínica asociada al acontecimiento estresante vivido (ver Tabla 3), se observó que ninguna de las VP+ predecía el cambio clínico en el post-tratamiento ni en el seguimiento de los 3 meses. Sin embargo, se encontró que haber puntuado más bajo en la escala de crecimiento personal y autoaceptación del BP-29 predijo un mayor cambio en el IEP a los 6 y 12 meses, respectivamente, tras la intervención.

Tabla 3

Resultados del análisis de regresión entre VP+ y el cambio clínico en el IEP

\begin{tabular}{llcccc}
\hline IEP & & $\mathrm{R}^{2}$ & $\beta$ & $\mathrm{t}$ & $\rho$ \\
\hline Pre-seg6 & BP-29: Crecimiento personal &, 16 & $-0,44$ & $-2,36$ &, $027^{*}$ \\
Pre-seg12 & BP-29:Autoaceptación &, 59 & $-0,81$ & $-3,33$ &, $016^{*}$ \\
\hline
\end{tabular}

${ }^{* *} \rho<0,01 ;{ }^{*} \rho<0,05$

Por último, los resultados referidos al cambio clínico en la escala PANAS se presentan en las Tablas 4 y 5 . En cuanto al cambio clínico en el afecto positivo (Tabla 4), ninguna de las $\mathrm{VP}+$ resultó ser predictora del cambio tras la intervención ni al año de finalizar el tratamiento. Sin embargo, en el seguimiento de los 3 meses, se encontró que haber puntuado más alto en la autoaceptación y más bajo en autoestima predijo un mayor cambio clínico. Mientras que en el seguimiento de los 6 meses, un mayor cambio en afecto positivo se observó en personas que habían puntuado más bajo en EROS.

En los resultados obtenidos para la escala de afecto negativo se observó que haber puntuado más alto en autonomía antes del tratamiento predecía un mayor cambio en esta medida en el post-tratamiento y el seguimiento a los 3 meses. Nuevamente, en el seguimiento de los 6 meses se observó un mayor cambio en las personas que percibían su entorno menos reforzante en la fase de pre-tratamiento. 
Tabla 4

Resultados del análisis de regresión entre las VP+ y el cambio clínico en el afecto positivo

\begin{tabular}{llcccc}
\hline PANAS+ & & $\mathrm{R}^{2}$ & $\beta$ & $\mathrm{t}$ & $\rho$ \\
\hline Pre-seg3 & Autoestima &, 20 & 0,53 & 2,95 &, $006^{* *}$ \\
& BP-29: Autoaceptación & & $-0,37$ & $-2,05$ &, $050^{*}$ \\
Pre-seg6 & EROS &, 14 & 0,42 & 2,32 &, $029^{*}$ \\
\hline
\end{tabular}

${ }^{* *} \rho<0,01 ;{ }^{*} \rho<0,05$

Tabla 5

Resultados del análisis de regresión entre las VP+ y el cambio clínico en el afecto negativo

\begin{tabular}{llcccc}
\hline PANAS- & & $\mathrm{R}^{2}$ & $\beta$ & $\mathrm{t}$ & $\rho$ \\
\hline Pre-post & BP-29:Autonomía &, 13 & 0,38 & 2,59 &, $014^{* *}$ \\
Pre-seg3 & BP-29: Autonomía &, 26 & 0,53 & 3,46 &, $002^{* *}$ \\
Pre-seg6 & EROS &, 43 & $-0,67$ & $-4,59$ &, $000^{* *}$ \\
\hline
\end{tabular}

${ }^{* *} \rho<0,01 ;{ }^{*} \rho<0,05$

\section{Discusión}

El objetivo del presente trabajo fue analizar, por un lado, la capacidad de algunas VP+ para predecir la intensidad de la sintomatología clínica en los TA y, por otro, explorar en qué medida dichas variables predecían también el cambio clínico tras la intervención. Los resultados obtenidos según los análisis de regresión lineal indican que las $\mathrm{VP}+$, tanto personales como del entorno, funcionan como factores protectores ante la adversidad en pacientes con TA. Estos resultados van en la misma línea que los hallados en otros trabajos y que sugieren que la respuesta a las situaciones estresantes depende de muchos factores, entre ellos las características de personalidad, el apoyo social y las estrategias de afrontamiento (Wilhelm, Wedgwood, Parker, Geerligs, y Hadzi-Pavlovic, 2010). Así, en el presente estudio se encontró que los pacientes que percibían su vida y sus actividades como más satisfactorias, presentaban una sintomatología clínica menos intensa al vivir una situación adversa. Por lo tanto, los resultados apoyan la utilidad de promover en las personas el desarrollo de rasgos, fortalezas y estrategias relacionados con la calidad de vida así como el crecimiento y madurez personal. De esta forma, los individuos estarían más preparados para superar las distintas situaciones de crísis actuales y futuras.

Otro hallazgo importante del trabajo indica que el hecho de poseer en menor medida los rasgos y características positivas mencionadas en el apartado anterior no impide a los pacientes beneficiarse del tratamiento para su problema. De hecho, se observó que los pacientes que presentaban en menor medida rasgos positivos, tales como autoestima, autoaceptación, percepción de evolución y motivación para el crecimiento personal, o percibían su entorno y sus actividades como menos reforzantes, experimentaban un mayor cambio clínico después de la intervención. Esto podría indicar que durante la intervención los pacientes adquieren co- 
nocimientos y herramientas necesarias para el cambio, independientemente de sus recursos iniciales. En este punto cabe destacar que el TCC recibido por los pacientes no se limitaba únicamente a reducir la sintomatología clínica, sino también a promover el cambio de actitud hacia los problemas, para así aprender de ellos y poder evolucionar. Por tanto, se trata de un programa de tratamiento que integra el enfoque de psicología clínica tradicional, cuyo objetivo es reducir el malestar, con el de psicología positiva, que busca el aumento de bienestar y el desarrollo de fortalezas humanas. Este carácter integrador podría ser clave para el cambio clínico y para prevenir la reaparición de futuros problemas psicológicos (Fava y cols., 2005).

Por último, los resultados revelaron que una mayor sensación de dominio o control del entorno y una mayor sensación de autonomía respecto a la presión social se asociaban a una mayor reducción de la sintomatología depresiva y afecto negativo, respectivamnete. Por tanto, nuevamente resulta evidente la necesidad de desarrollar programas que promuevan el bienestar y proporcionen recursos para afrontar las dificultades y alcanzar las metas personales. En la literatura se pueden encontrar diferentes propuestas para incrementar el bienestar en adolescentes (van Genugten, Dusseldor, Massey, y van Empelen, 2017), jovenes universitarios (Fernandez y cols., 2016), y adultos (Ivandic, Freeman, Birner, Nowak y Sabariego, 2017). Sin embargo, los resultados sobre su eficacia no son del todo concluyentes y hacen falta más estudios, de mayor rigurosidad científica, para poder identificar las mejores estrategias de prevención.

Finalmente, es importante señalar que el trabajo presenta limitaciones. Por ello, como propuestas de mejora para este estudio se proponen, por un lado, el aumento de la muestra, ya que el número total de sujetos con los que contamos para este trabajo fue bastante limitado y, por otro lado, utilizar un indicador de cambio clínico más riguroso, por ejemplo el índice de cambio fiable de Jacobson y Truax (1991). A pesar de estas limitaciones, los resultados de este trabajo suponen un avance tanto en el área de conocimiento de los TA como de Psicología Positiva.

\section{Referencias bibliográficas}

American Psychiatric Association. (APA) (2013). Diagnostic and statistical manual of mental disorders (5th Ed). Washington, DC: American Psychological Association.

Armento, M.E.A. y Hopko, D.R. (2007). The Environmental Reward Observation Scale (EROS): Development, validity, and reliability. Behavior Therapy, 38, 107-119.

Baños, R. M., Guillén, V., Botella, C., García, A., Jorquera, M., y Quero, S. (2008). Un programa de tratamiento para los trastornos adaptativos. Un estudio de caso. Apuntes de Psicología, 26(2), 303-316.

Barraca, J. y Pérez-Álvarez, M. (2010). Adaptación española del Environmental Reward Observation Scale (EROS). Ansiedad y Estrés, 16(1), 95-107.

Beck, A.T., Steer, R.A., y Brown, G.K. (1996). Manual for the Beck Depression Inventory-Second Edition. San Antonio, TX: The Psychological Corporation.

Berenbaum, H., y Oltmanns, T. F. (1992). Emotional experience and expression in schizophrenia and depression. Journal of Abnormal Psychology, 101(1), 37-44.

Botella, C., Baños, R.M., y Guillén, V. (2008). Una propuesta de tratamiento para los trastornos adaptativos: creciendo en la adversidad. En C. Vázquez y G. Hervás (Eds.), Psicología Positiva aplicada (pp. 129-154). Bilbao: Declée de Brouwer.

Calvo, E. (2009). Duración de la incapacidad temporal asociada a diferentes patologías en trabajadores españoles. Recuperado de http://www.seg-social.es/prdi00/groups/public/ documents/binario/146662.pdf 
Pressman, S. D. y Cohen, S. (2012). Positive emotion word use and longevity in famous deceased psychologists. Health Psychology, 31(3), 297-305.

Quero, S., Andreu-Mateu, S., Moragrega, I., Baños, R.M., Molés, M., Nebot, S, y Botella, C. (2017). Un programa cognitivo-conductual que utiliza realidad virtual para el tratamiento de los trastornos adaptatives: una serie de casos. Revista Argentina de Clínica Psicológica, 26(1), 5-18.

Quero, S., Molés, M., Mor, S., Baños, R., y Botella, C. (2014, noviembre). Spanish Validation of Complicated Grief Inventory adapted for Adjustment Disorder. Póster presentado en ABCT's 48th Annual Convention. Philadelphia.

Rosenberg, M. (1965). Society and the adolescent self-image. Princeton, NJ: Princeton University Press.

Ryff, C.D. (1989). Happiness is everything, or is it? Explorations on the measuring of research on hedònic and eudaimonic well-being. Annual Review of Psychology, 52, 141-166.

Sanz, J., García-Vera, M.P., Espinosa, R., Fortún, M., y Vázquez, C. (2005). Adaptación española del Inventario para la Depresión de Beck-II (BDI-II): Propiedades psicométricas en pacientes con trastornos psicológicos. Clínica y Salud, 16(2), 121-142.

Sanz, J., Perdigón, A.L., y Vázquez, C. (2003). Adaptación española del Inventario para la Depresión de Beck-II (BDI-II): Propiedades psicométricas en población general. Clínica y Salud, 14(3), 249-280.

Schmitt, D.P. y Allik, J. (2005). Simultaneous administration of the Rosenberg Self-Esteem Scale in 53 nations: explorin the universal and culture-specific features of global self-esteem. Journal of Personality and Social Psychology, 89(4), 623-642.

Seligman, M.E., Steen, T. A., Park, N., y Peterson, C. (2005). Positive psychology in progress. Empirical validation of interventions. American psychologist, 60, 410-421.

Sheung-Tak, C., Emily, P. M., Helene, H., Timothy, L., Lee, D., y Linda, C. W. (2017). Benefitfinding and effect on caregiver depression: A double-blind randomized controlled trial. Journal of Consulting and Clinical Psychology, 85(5), 521-529.

Sloan, D., Strauss, M. E., y Wisner, K. (2001). Diminished response to pleasant stimuli by depressed women. Journal of Abnormal Psycology, 110(3), 488-493

Tseferidi, S. I., Griva, F., y Anagnostopoulos, F. (2017). Time to get happy: associations of time perspective with indicators of well-being. Psychology, health y medicine, 22(5), 618-624.

Van Genugten, L., Dusseldorp, E., Massey, E.K., y van Empelen, P. (2016). Effective selfregulation change techniques to promote mental wellbeing among adolescents: a metaanalysis. Health Psychology Review, 11(1), 53-71.

Watson, D., Clark, L.A., y Tellegen, A. (1988). Development and validation of brief measures of positive and negative affect: the PANAS Scales. Journal of Personality and Social Psychology, 54(6), 1063-1070.

Watson, D., Stasik, S.M., Ellickson-Larew, S., y Stanton, K. (2015). Extraversion and psychopathology: A facet-level analysis. Journal of Abnormal Psychology, 124, 432-446.

Wilhelm, K., Wedgwood, L., Parker, G., Geerligs, L., y Hadzi-Pavlovic, D. (2010). Predicting mental Health and well-being in adulthood. The Journal of Nervous and Mental Disease, 198(2), 85-90. 
Carta, M. G., Balestrieri, M., Murru, A., y Hardoy, M. C. (2009). Adjustment Disorder: epidemiology, diagnosis and treatment. Clinical Practice and Epidemiology in Mental Health, $5(15)$.

Cassey, P., Dowrick, C., y Wilkinson, G. (2001). Adjustment disorders. Fault line in the pychiatric glossary. The British Journal of Psychiatry, 179, 479-481.

Cassey, P., Jabbar, F., O'Leary, E., y Doherty, A.M. (2015). Suicidal behaviours in adjustment disorder and depressive episode. Journal of Affective Disorders, 174, 441-446.

Charles, T., Lyubomirsky, S., y Murray, B. (2017). Upregulating the positive affect system in anxiety and depression: outcomes of a positive activity intervention. Depression and anxiety, 34(3), 267-280.

Clark, L. A. (2005). Temperament as a unifying basis for personality and psychopathology. Journal of Abnormal Psychology, 114, 505-521.

Clark, L. A. y Watson, D. (1991). Tripartite model of anxiety and depression: Psychometric Evidence and taxonomic implications. Journal of Abnormal Psychology, 100, 316-336.

Davidson, R.J. (1998). Affective style and affective disorders: Perspectives from affective neuroscience. Cognition and Emotion, 12, 307-330.

Díaz, D., Rodríguez-Carvajal, R., Blanco, A., Moreno-Jiménez, B., Gallardo, I., Valle, C., y van Dierendonck, D. (2006). Adaptación española de las escalas de bienestar psicológico de Ryff. Psicothema, 18(3), 572-577.

Fava, G.A., Ruini, C., Rafanelli, C., Finos, L., Salmaso, L., Mangelli, L., y Sirigatti, S. (2005). Psychotherapy and Psychosomatics, 74(1), 26-30.

Fernandez, A., Howse, E., Rubio-Valera, M., Thorncraft, K., Noone, J., Luu, X., Veness, B., Leech, M., Llewellyn, G., y Salvador-Carulla, L. (2016). Setting-based interventions to promote mental health at the university: a systematic review. International Journal of Public Health, 61(7), 797-807.

Fredrickson, B. L. (1998). What good are positive emotions?. Review of General Psychology, 2(3), 300-319

Ivandic, I., Freeman, A., Birner, U., Nowak, D., y Sabariego, C. (2017). A systematic review of brief mental health and well-being interventions in organizational settings. Scandinavian Journal of Work, Environment y Health, 43(2), 99-108.

Jacobson, N.S. y Truax, P. (1991). Clinical significance: A statistical approach to defining meaningful change in psychotherapy research. Journal of Consulting and Clinical Psychology, 59(1), 12-19

Johnson, M. D., Galambos, N. L., Finn, C., Neyer, F. J., y Horne, R. M. (2017). Pathways between self-esteem and depression in couples. Development psychology, 53(4), 787-799.

Kaviani, H., Gray, J. A., Checkley, S. A., Raven, P. W., Wilson, G. D., y Kumari, V. (2004). Affective modulation of the startle response in depression: Influence of the severity of depression, anhedonia, and anxiety. Journal of Affective Disorders, 83(1), 21-31.

Klein, H. A. (1974). Behavior modification as therapeutic paradox. American Journal of Orthopsychiatry, 44(3), 353-361.

Krueger, R. F., McGue, M., y lacono, W. G. (2001). The higher-order structure of common DSM mental disorders: Internalization, externalization, and their connections to personality. Personality and Individual Differences, 30, 1245-1259.

Küçük, E. E. y Küçük-Alemdar, D. (2017). Life satisfaction and psychological status of mothers with disabled children: a descriptive study. Community mental health journal. doi:10.1007/ s10597-017-0135-6

Li, Y.I., Starr, L.R., y Hershenberg, R. J. (2017). Responses to positive affect in daily life: positive rumination and dampening moderate the association between daily events and depressive symptoms. Journal of Psychopathology Behavioral Assessment, 1-14. 